\title{
Quantitative CT perfusion imaging in patients with pancreatic cancer: a systematic review
}

\author{
T. H. Perik ${ }^{1}$ (I) - E. A. J. van Genugten ${ }^{1}$ - E. H. J. G. Aarntzen ${ }^{1}$ - E. J. Smit ${ }^{1}$ - H. J. Huisman ${ }^{1}$ · J. J. Hermans ${ }^{1}$
}

Received: 16 April 2021 / Revised: 18 June 2021 / Accepted: 21 June 2021 / Published online: 5 July 2021

(c) The Author(s) 2021

\begin{abstract}
Pancreatic ductal adenocarcinoma (PDAC) is the third leading cause of cancer-related death with a 5-year survival rate of 10\%. Quantitative CT perfusion (CTP) can provide additional diagnostic information compared to the limited accuracy of the current standard, contrast-enhanced CT (CECT). This systematic review evaluates CTP for diagnosis, grading, and treatment assessment of PDAC. The secondary goal is to provide an overview of scan protocols and perfusion models used for CTP in PDAC. The search strategy combined synonyms for 'CTP' and 'PDAC.' Pubmed, Embase, and Web of Science were systematically searched from January 2000 to December 2020 for studies using CTP to evaluate PDAC. The risk of bias was assessed using QUADAS-2. 607 abstracts were screened, of which 29 were selected for full-text eligibility. 21 studies were included in the final analysis with a total of 760 patients. All studies comparing PDAC with non-tumorous parenchyma found significant CTP-based differences in blood flow (BF) and blood volume (BV). Two studies found significant differences between pathological grades. Two other studies showed that BF could predict neoadjuvant treatment response. A wide variety in kinetic models and acquisition protocol was found among included studies. Quantitative CTP shows a potential benefit in PDAC diagnosis and can serve as a tool for pathological grading and treatment assessment; however, clinical evidence is still limited. To improve clinical use, standardized acquisition and reconstruction parameters are necessary for interchangeability of the perfusion parameters.
\end{abstract}

\section{Graphic abstract}

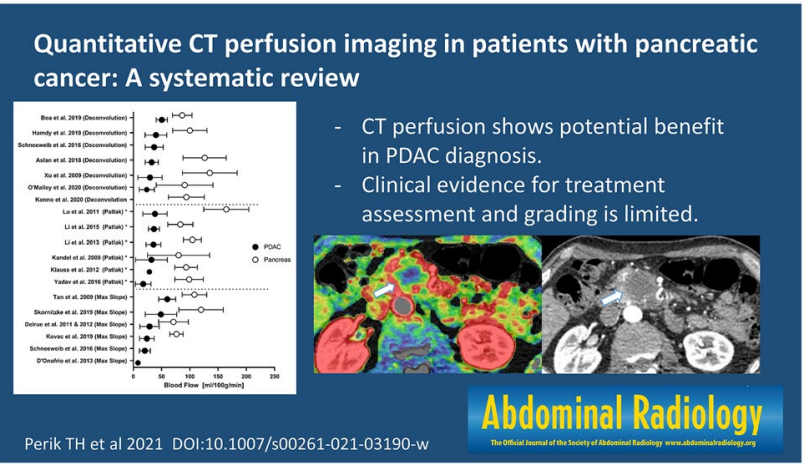

Keywords CT perfusion · Adenocarcinoma · Pancreas · Quantitative imaging

\section{Introduction}

T. H. Perik

tom.perik@radboudumc.nl

1 Department of Medical Imaging, Radboud University Medical Center, P.O. Box 9101, 6500 HB Nijmegen, The Netherlands
Pancreatic ductal adenocarcinoma (PDAC) is the third leading cause of cancer-related death in the USA [1]. The incidence increases by an estimated $0.5 \%$ per year, while prognosis remains poor, with a 5 -year survival rate of $10 \%$ 
[1]. Availability and advancement of imaging technology and new treatment options have not improved 5-year survival in the last 40 years, mainly due to late presentation [2]. As $40 \%$ presents with locally advanced disease and $40-45 \%$ with metastatic disease, the majority of patients face incurable disease [3].

Biphasic or triphasic contrast-enhanced CT (CECT), with at least both an arterial and portal-venous phase, is the current standard for diagnosis, assessment of resectability, and monitoring of therapy of PDAC [4]. However, several problems are currently encountered using CECT. First, tumor characterization and delineation, essential for staging and pre-operative planning, are not always possible. Accurate diagnosis based on CECT is difficult as tumors may appear isoattenuating to the surrounding parenchyma in $15-20 \%$ of cases $[5,6]$. In addition, inflammatory masses of acute and chronic pancreatitis can mimic PDAC on CECT, risking misdiagnosis [7]. Second, histopathological analysis is the gold standard, and tumor grading is an important parameter of survival [8]. However, biopsies do not always yield sufficient material for pathological grade analysis. With fineneedle biopsy (FNB), accurate histology is retrieved in $77 \%$ of all procedures, at the risk of complications [9]. Furthermore, as PDAC is a heterogeneous tumor, biopsy could lead to a sampling error [10]. A noninvasive, reliable imaging biomarker would be highly desirable to accurately assess the histological grading of the complete tumor, which could aid in selecting patients for the appropriate treatment. Third, with the current imaging techniques, RECIST-based treatment assessment is insufficient. Neoadjuvant chemotherapy is increasingly applied for potential resectable PDAC [11]. However, RECIST-based restaging remains troublesome because changes in tumor size and vessel encasement using CECT prove to be inadequate for reliable response assessment after neoadjuvant treatment [12]. Moreover, the correlation between RECIST and histopathological grading of tumor response is poor [13]. Thus, CECT response criteria could underestimate the treatment response, showing the need for a more reliable predictor to increase the amount of margin-negative resections (i.e., R0).

CT perfusion (CTP), in other words dynamic contrastenhanced CT, is a novel modality that could improve the diagnostic workup of PDAC by combining functional information and spatial detail [14]. CTP is an imaging technique where dynamic acquisition after injection of a contrast agent enables quantification of tissue vascularization [15]. Using a kinetic model, parameters can be calculated, which reflect intratumoral differences in perfusion and vascular permeability. Therefore, CTP bears potential as a biomarker in oncology for tumor angiogenesis, enabling prediction of tumor grading and assessment of treatment response [16-19]. Over the last years, CTP is increasingly utilized as a functional imaging biomarker, and several articles reported additional benefits and advances of CTP in pancreatic cancer.

This systematic review aims to evaluate the literature on CTP in pancreatic cancer for diagnosis, grading, and treatment assessment. The secondary goal is to provide an overview of scan protocols and perfusion models used for CTP in the pancreas.

\section{Materials and methods}

\section{Search strategy}

This systematic review was carried out in accordance with the Preferred Reporting Items for Systematic Reviews and Meta-Analysis 2015 (Prisma-P 2015) [20]. The protocol for this systematic review is registered in the PROSPERO database [Registration number: CRD42021213438] [21]. The search strategy combined synonyms for 'CTP', 'Dynamic contrast-enhanced CT,' 'Pancreatic cancer,' and 'Pancreatic adenocarcinoma'; the complete search is accessible in the Supplementary materials. This systematic search was performed in the following libraries: PubMed, EMBASE, and Web of Science; search terms were tailored to each database. The timeframe for published articles was 1 January 2000-31 December 2020.

\section{Eligibility criteria}

Studies were considered eligible when CTP was used during diagnosis or treatment assessment of primary PDAC. The scan protocol must be described clearly and consists of dynamic acquisitions resulting in calculated perfusion parameters, such as blood flow (BF), blood volume (BV), and permeability surface area product (PS).

\section{Study selection}

Retrieved articles were imported into EndNote and duplicates removed. The article titles and abstracts resulting from the search were screened independently by two reviewers (T.P, E.G.). Studies meeting the inclusion criteria were selected for full-text screening and reviewed. In the subsequent full-text screening stage, any disagreements were resolved by consensus, and if consensus was not reached, a third reviewer (J.H.) was consulted.

\section{Data extraction/synthesis}

Relevant study characteristics and scan parameters were extracted by one reviewer and checked by the second reviewer. Scan parameters included type of detector, number of acquisitions, contrast injection, and type of kinetic 
model. Comparison of studies was performed using perfusion values such as BF, BV, and PS. To compare different studies, perfusion parameters were converted to $\mathrm{mL} / 100 \mathrm{~g} /$ min when reported in $\mathrm{mL} / 100 \mathrm{~mL} / \mathrm{min}$ using a tissue density of $1.05 \mathrm{gr} / \mathrm{mL}$ [15]. Due to heterogeneity in the data, only descriptive statistic were applied. Studies were categorized based on the goal of the study and the type of kinetic model. Graphs were created using GraphPad Prism 9.

\section{Quality assessment}

The risk of bias and applicability of each study were assessed using the Quality Assessment of Diagnostic Accuracy Studies (QUADAS-2) tool. The risk of bias and applicability concerns is defined as the risk to deviate from the QUADAS-2 guidelines described in four domains: patient selection, index test, reference standard, and flow and timing. QUADAS signal questions regarding index test were adjusted to classify description of perfusion scan protocol, kinetic model software, and ROI measurement. Consensus about the assessment was reached between the same two reviewers who selected studies for inclusion.

\section{Results}

\section{Study selection}

With the described search strategy, 881 articles were identified, which were reduced to 607 articles after removing duplicates. 607 articles were screened by title and abstracts, resulting in 29 articles eligible for full-text screening. Eight full-text articles were excluded as they did not meet the final inclusion criteria. Finally, 21 articles were included in the qualitative analysis, with a total of 760 patients with PDAC. All included studies with scan parameters and kinetic models can be found in Table 1, and study characteristics can be found in Table 2 .

The Prisma-2015 flowchart describing the selection process is visible in Fig. 1.

\section{Diagnosis}

In 15 out of 21 studies, BF was measured in both PDAC and non-tumorous pancreatic parenchyma, including a total of 519 patients. In all these studies, mean blood flow was significantly lower in tumor tissue compared to pancreatic parenchyma outside the tumor or in healthy pancreatic tissue in a control group [22-37]. Mean BF ranged from 17 to $60 \mathrm{~mL} / 100 \mathrm{~g} / \mathrm{min}$ for PDAC and $71-164 \mathrm{~mL} / 100 \mathrm{~g} / \mathrm{min}$ for pancreatic parenchyma. All mean BF values can be found in Fig. 2.
In 11 studies, BV was measured for PDAC and nontumorous pancreatic parenchyma for a total of 423 patients. [22-24, 26, 30, 32, 33, 35-38]. In all of these studies a statistically significantly lower mean BV was found for PDAC compared to pancreatic parenchyma outside the tumor. Mean BV ranged from 2.8 to $59 \mathrm{~mL} / 100 \mathrm{~g}$ for PDAC and $15-200 \mathrm{~mL} / 100 \mathrm{~g}$ for pancreatic parenchyma. All mean BV values can be found in Fig. 3 .

In 9 studies, permeability surface product (PS) was measured for both PDAC and non-tumorous pancreatic parenchyma in a total of 349 patients. In all these studies, the mean permeability was lower in pancreatic tumor tissue [22, $23,25,26,30,32,33,35,36]$. In two studies, this difference was statistically significant [22, 33]. Mean PS for PDAC ranged from 11 to $38 \mathrm{~mL} / 100 \mathrm{~g} / \mathrm{min}$ and for non-tumorous pancreatic parenchyma from 20 to $56 \mathrm{~mL} / 100 \mathrm{~g} / \mathrm{min}$. All mean PS values can be found in Fig. 4.

Aslan et al. and Delrue et al. showed a decrease in perfusion (BF and $\mathrm{BV}$ ) for both chronic pancreatitis and PDAC. However, compared to acute and chronic pancreatitis, the $\mathrm{BF}$ and $\mathrm{BV}$ were significantly lower $(p<0.01)$ in PDAC, even for isoattenuating PDAC $[22,37]$. Yadav reported that 6/42 lesions isoattenuating on CECT were visible on perfusion color maps. For differentiating PDAC from pancreatitis Yadav et al. report an AUC of 0.83 for BF and 0.80 for BV [33]. Aslan reports both sensitivity and specificity of $100 \%$ for BF and BV by using optimal cutoff values [22].

Some studies also assessed semi-quantitative parameters such as peak enhancement and mean transit time (MTT). Peak enhancement showed in two studies to be significantly higher in pancreatic parenchyma than in PDAC (Lu: 30.8 vs $57 \mathrm{HU} p<0.016$, Tan: 59 vs $101 \mathrm{HU} p<0.001$ ) [31, 34]. In two other studies, MTT was significantly higher in PDAC compared to healthy pancreatic parenchyma [Aslan: $11.2 \mathrm{vs.}$ 3.7 s, $(p<0.001)$, Kovac: 7.4 vs. 4 s $(p<0.001)][22,24]$.

\section{Grading}

Three articles evaluate the use of CTP to predict histopathological grading of PDAC compared to histopathological diagnosis obtained using a tumor biopsy or resected specimen $[24,39,40]$. These three studies included a total of 124 patients $[24,39,40]$.

In two articles, tumors were classified into two groups: low grade (well or moderate differentiated) and high grade (poorly differentiated) [24, 40]. Both studies found higher $\mathrm{BV}$ in low-grade tumors compared to high-grade tumors ( $p=0.001$ and $p=0.004)$, whereas a significant difference in BF was only reported by one article $(p=0.041)$. Furthermore, peak enhancement intensity compared to baseline $\mathrm{HU}$ was significantly different between high-grade tumors and low-grade tumors, respectively, $16 \mathrm{HU}$ vs. $26 \mathrm{HU}$. Time to peak (TTP) did not show a significant difference between 


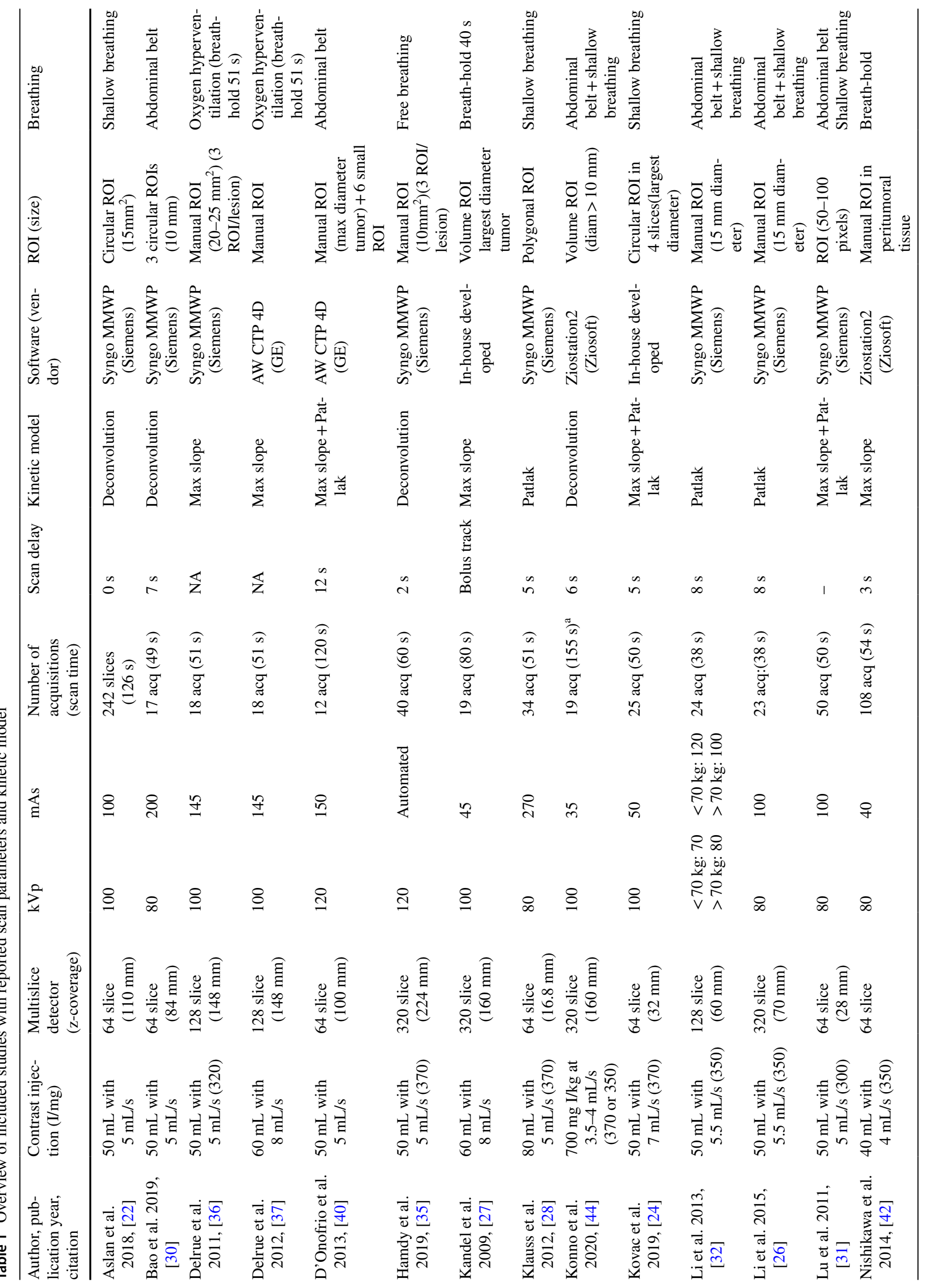




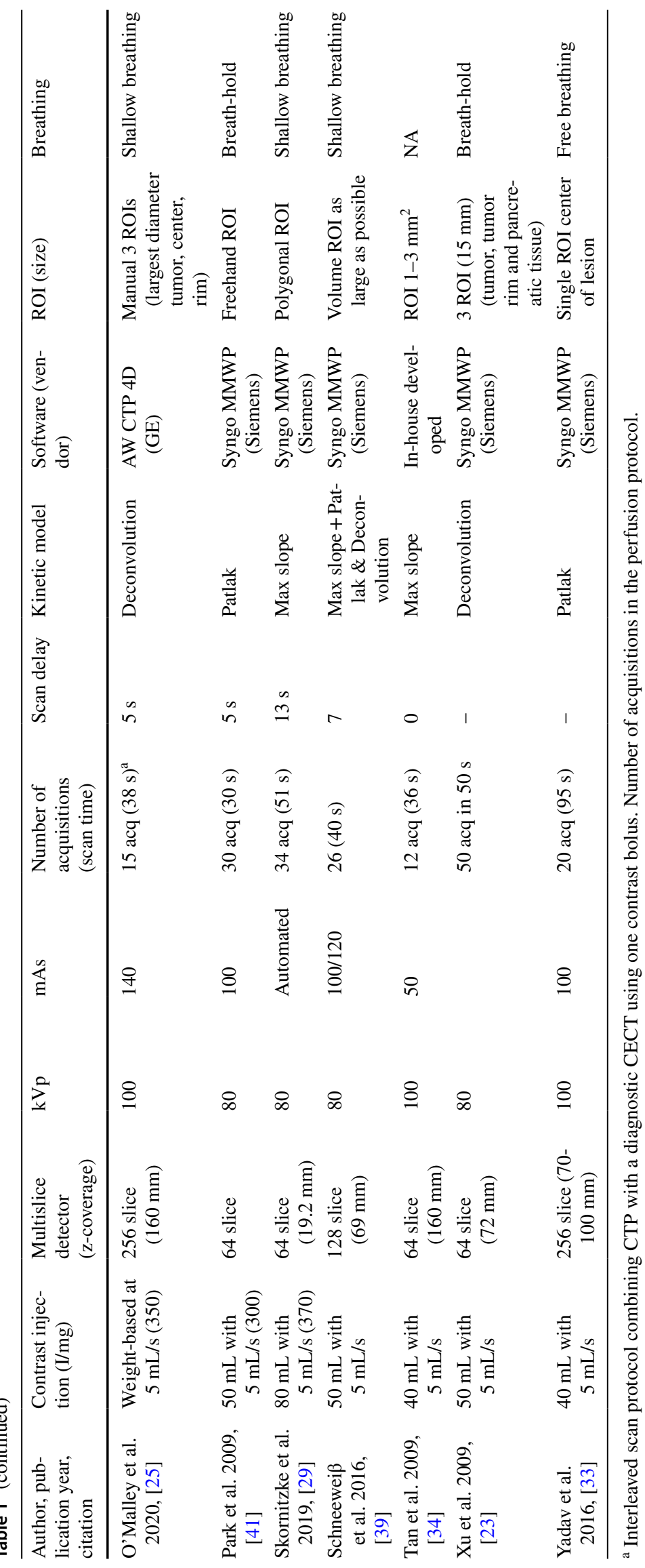




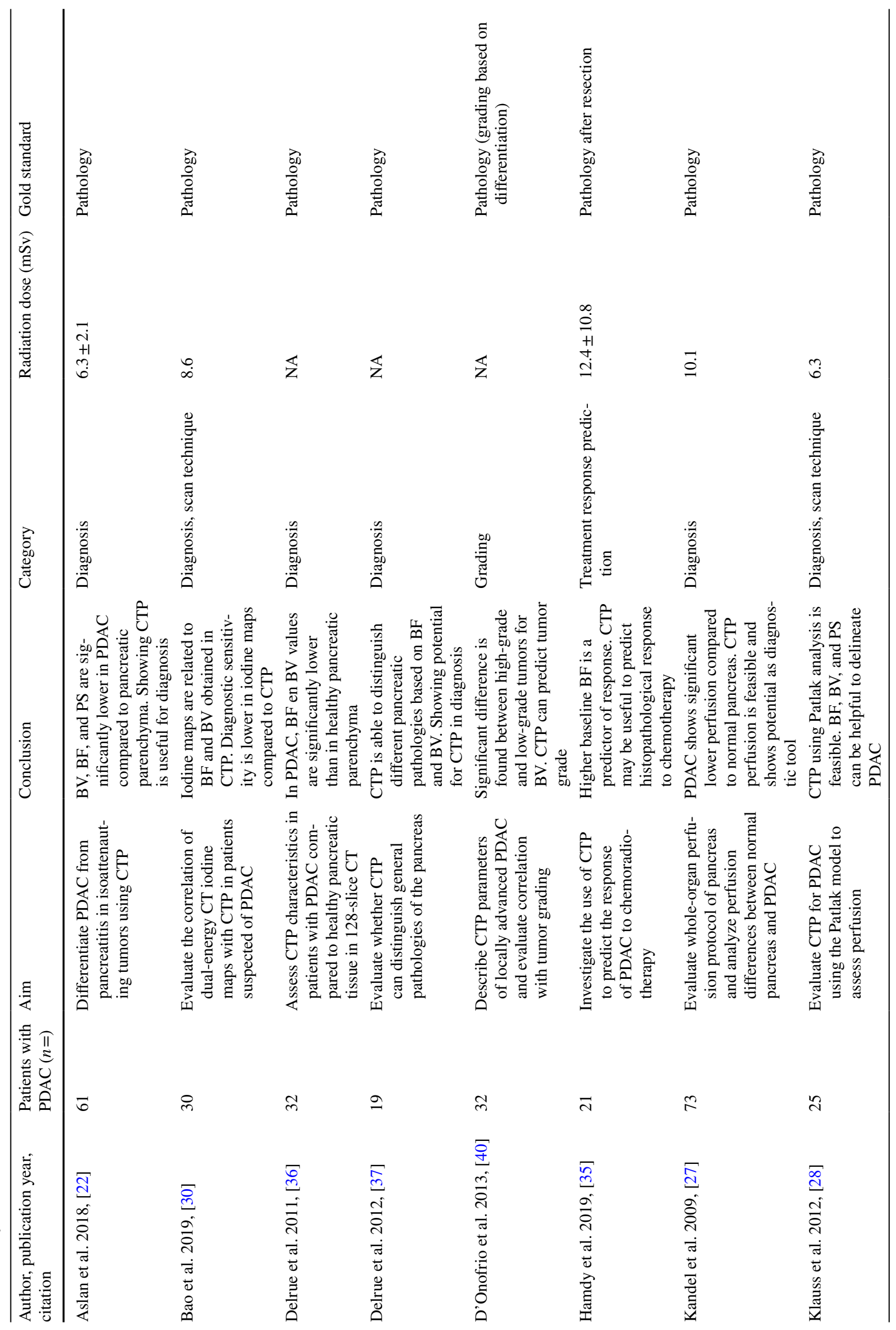




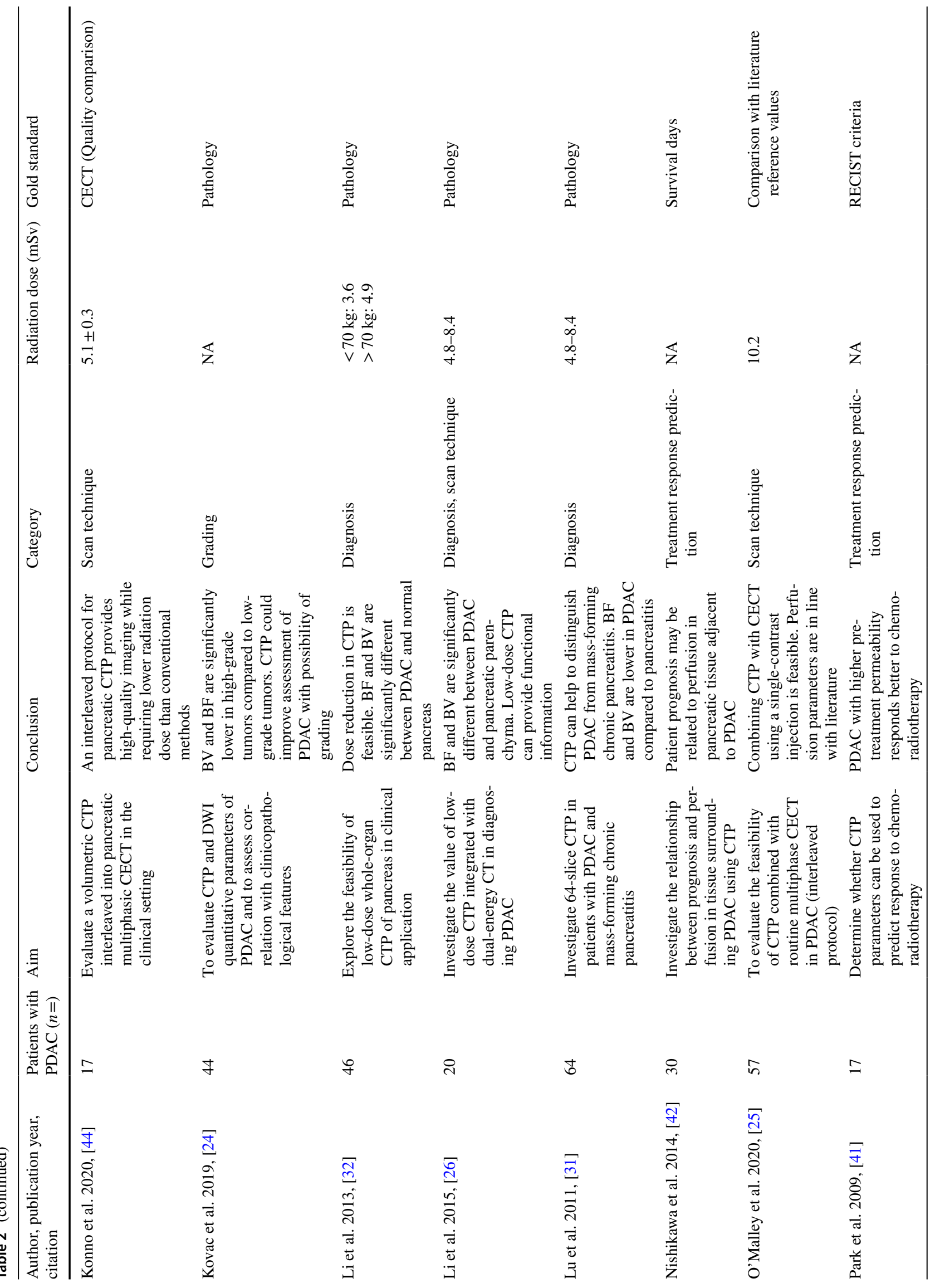




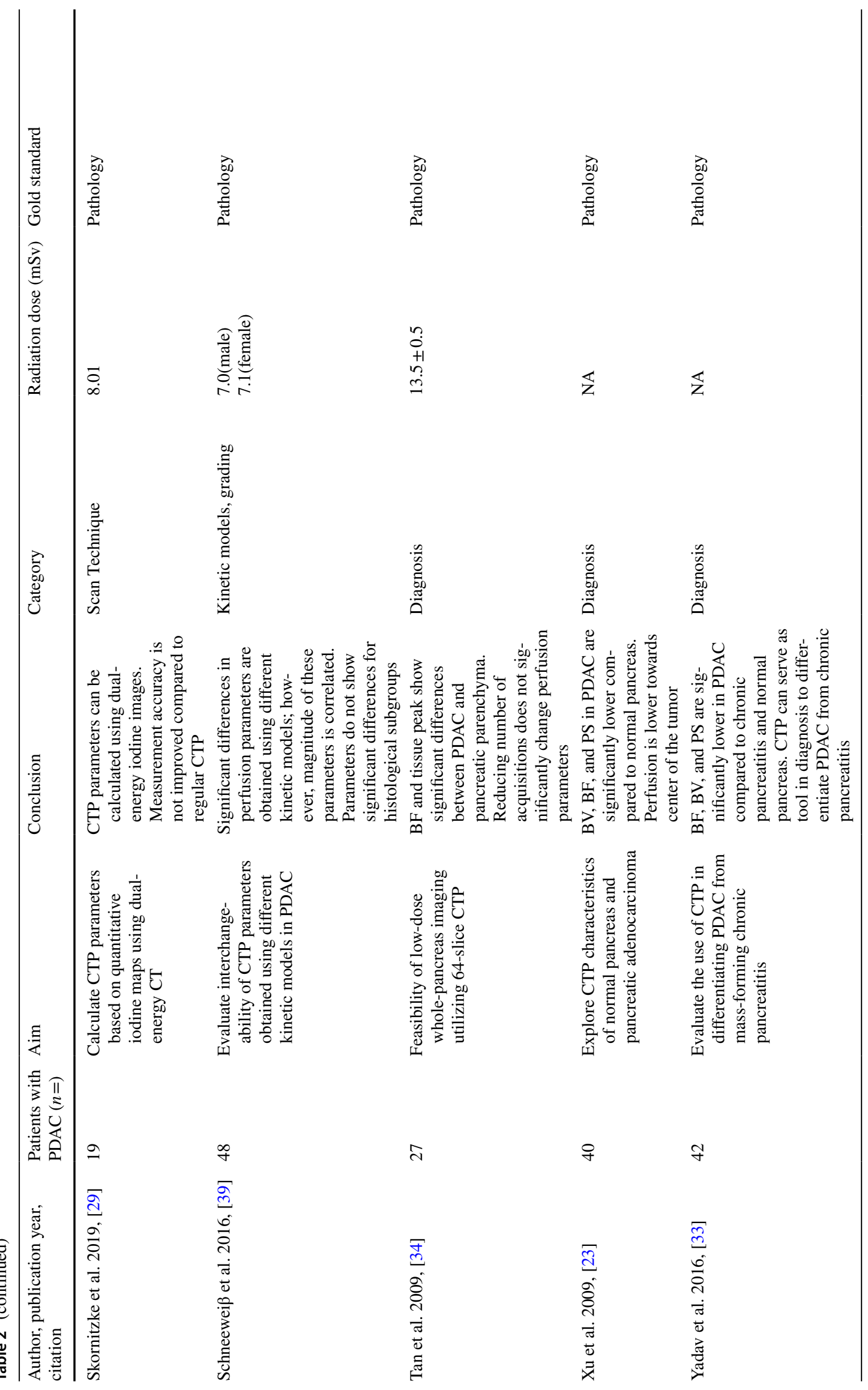


Fig. 1 Prisma-2015 flowchart of the study selection process

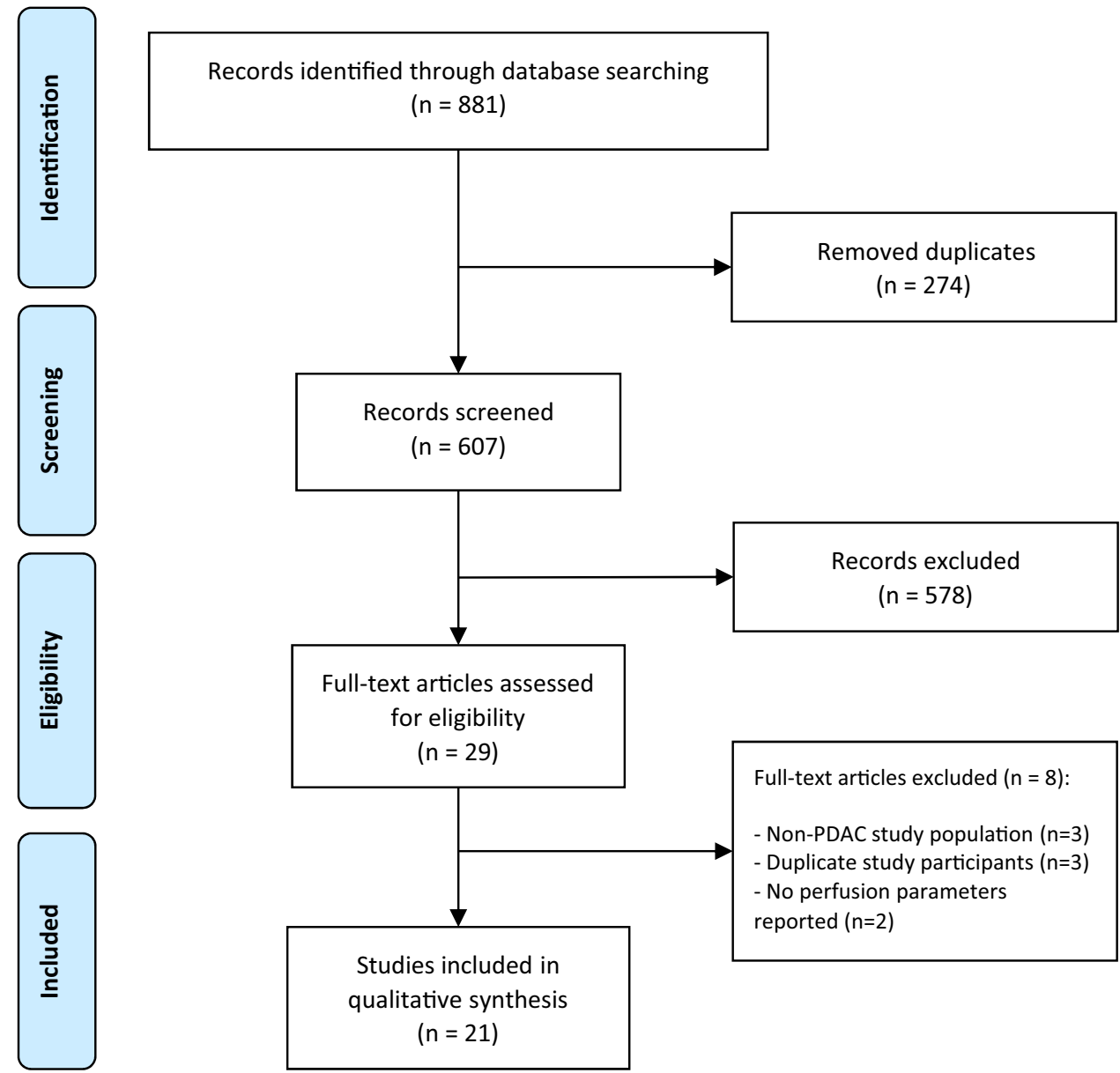

tumor grades. In addition, a ROC curve analysis was performed to assess the prediction of tumor grade; Kovac et al. calculated an AUC of 0.940 for BF and 0.977 for BV, while D'Onofrio et al. calculated an AUC of 0.798 for BV [24, 40]. Perfusion parameters for the different grades can be found in Table 3.

In the third study, tumors were graded in three groups: well differentiated, moderately differentiated, and poorly differentiated. Using both deconvolution and Patlak combined with Max slope to calculate BF, BV, and PS, no significant differences between the three groups of pathological grade were found [39].

\section{Treatment response prediction}

Two studies investigated the role of CTP as a predictor of treatment response to neoadjuvant chemoradiotherapy with a total of 38 included patients [35, 41]. In both studies, treatment included 45-50,4 Gy radiation in 25-28 fractions and combined gemcitabine-based chemotherapy. Hamdy et al. classified response based on histology of the resection specimen after chemoradiotherapy, whereas Park et al. used the RECIST criteria on conventional CECT after 3 months to assess treatment response; both studies performed CTP before treatment. Hamdy et al. reported a higher pre-treatment BF for responders compared to non-responders (44 vs. $28 \mathrm{~mL} / 100 \mathrm{~g} / \mathrm{min}, p=0.04)$, whereas PS values were similar [35]. Pre-treatment perfusion measurements of Park et al. showed a significantly higher permeability in responders compared to non-responders (50.8 vs. $19.0 \mathrm{~mL} / 100 \mathrm{~mL} /$ $\min , p=0.01$ ) [41]. In both studies, pre-treatment BV was higher in responders compared to non-responders, although not significant.

Hamdy et al. performed a follow-up CTP after chemoradiation therapy, 7 weeks after baseline CTP. Both BF $(p=0.04)$ and $\mathrm{BV}(p=0.01)$ increased significantly in responders after chemoradiotherapy compared to baseline CTP). In non-responders, a non-significant increase in BF $(p=0.06)$ and BV $(p=0.06)$ was reported [35]. Table 4 provides an overview of the perfusion parameters of responders and non-responders.

Instead of treatment response, another study performed a prediction of survival based on CTP. Perfusion values in peritumoral tissue directly adjacent to tumor tissue were assessed, resulting in a correlation between higher peritumoral blood flow and shorter survival $(p=0.004)$ [42]. 
Fig. 2 Mean and standard deviation of blood flow of tumor (PDAC) and non-tumorous pancreatic parenchyma in all studies sorted by kinetic model. $\mathrm{BF}$ in tumor tissue is lower compared to non-tumorous pancreas parenchyma in all studies. *Patlak model was reported in these studies. However, this model solely is not able to calculate BF

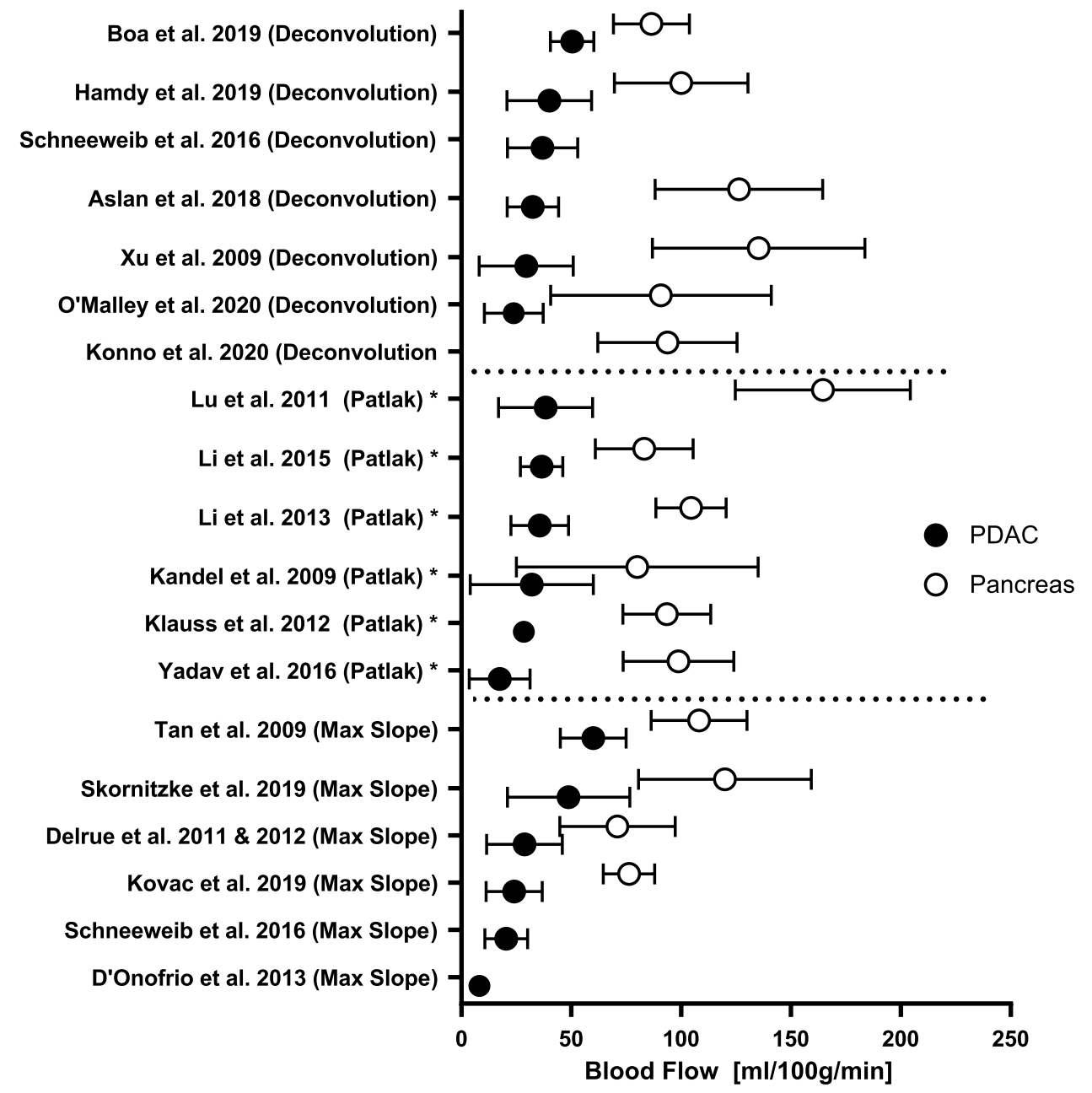

\section{Scanning protocols}

In five studies, the main goal was the evaluation of the CTP scan technique for pancreatic cancer. A novel scanning method of an interleaved CTP protocol, where the perfusion acquisition was interleaved with a diagnostic multiphase contrast CECT was introduced in 2013 [43]. This method requires only one contrast bolus, instead of two separate boluses for the perfusion scan and diagnostic scan, allowing for a 'one-stop-shop' approach [25, 43, 44]. Two studies demonstrated that simultaneous acquisition of perfusion and high-quality diagnostic images with a single contrast bolus was feasible without difference in quality compared to conventional CECT [25, 44].

Three studies focused on the use of dynamic dual-energy $\mathrm{CT}$ acquisitions to calculate perfusion parameters. Li et al. combined both techniques by performing a dual-energy CT after a CTP scan, using the time-attenuation curve to improve the timing of the dual-energy CT [26]. Skornitzke et al. calculated BF based on DECT iodine enhancement images. However, these CTP maps did show a significant improvement compared to conventional acquisitions [29].
Bao et al. showed a good correlation of iodine concentration with both $\mathrm{BF}$ and $\mathrm{BV}$, indicating the potential of dual-energy $\mathrm{CT}$ to reflect hemodynamic changes using a lower radiation dose [30]. However, the sensitivity to discriminate PDAC from non-tumorous pancreatic parenchyma was higher using CTP parameters with an AUC of 0.971 and 0.958 for BF and $\mathrm{BV}$, compared to AUC of 0.842 for dual-energy-based iodine maps.

\section{Kinetic models}

In the reviewed articles, calculation of perfusion parameters is performed using three main kinetic models: Max slope (one compartment), Patlak (two compartment), and deconvolution.

The maximum slope model assumes a single compartment to estimate the BF using the maximum slope of the time-attenuation curve. In addition, semi-quantitative parameters can be deduced from this time-attenuation curve, like peak enhancement compared to baseline and time to peak [45]. The single-compartment model assumes the 

ation of blood volume of tumor (PDAC) and non-tumorous pancreatic parenchyma in all studies sorted by kinetic model. $\mathrm{BV}$ in tumor tissue is lower compared to non-tumorous pancreas parenchyma in all studies. *Maximum slope model was reported in these studies. However, this model solely is not able to calculate BV
Fig. 3 Mean and standard devi-
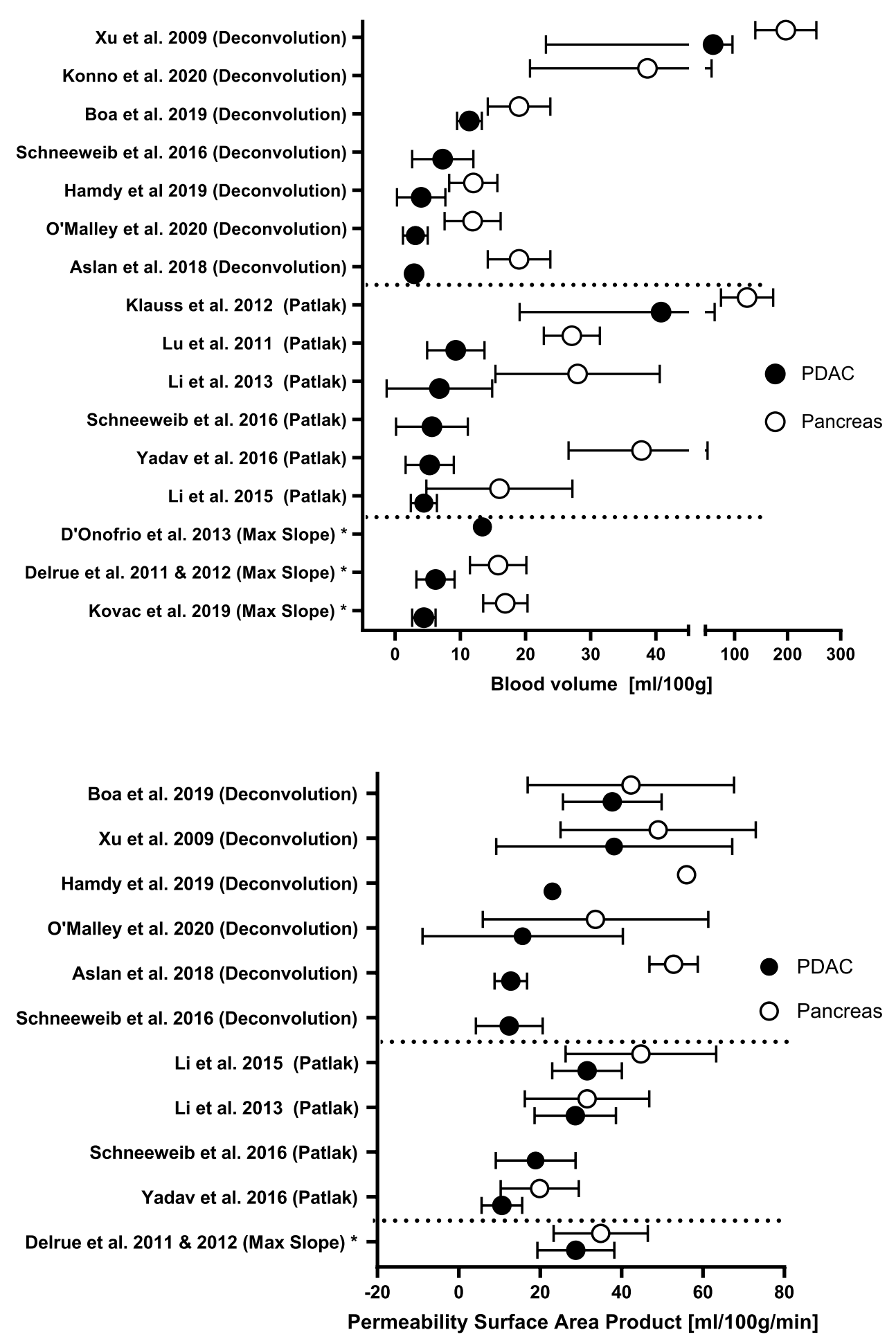

Fig. 4 Mean and standard deviation of vascular permeability surface area product (PS) of tumor (PDAC) and non-tumorous pancreatic parenchyma in studies sorted by kinetic model. *Maximum slope model was reported in these studies. However, this model solely is not able to calculate PS

absence of venous outflow, therefore perfusion parameters as BV, and PS cannot be estimated [46].

The standard Patlak plot is a linear graphical representation of a two-compartment model, which assumes the distribution of injected contrast agent over two well-mixed compartments. The model is based on an irreversible transfer of contrast agent from the intravascular to the extravascular compartment, allowing estimations of the blood volume and permeability based on the linear part of the Patlak plot [47-49].

In the deconvolution method, the time-attenuation curve of the tissue is assumed to be the convolution between the arterial input function and the impulse residue function. This last curve is a theoretical representation of 
Table 3 For different histopathological grading of PDAC mean/median, BF values are reported in $(\mathrm{mL} / 100 \mathrm{~g} /$ $\mathrm{min}), \mathrm{BV}$ is reported in $(\mathrm{mL} / 100 \mathrm{~g})$, and PS is reported in $(\mathrm{mL} / 100 \mathrm{~g} / \mathrm{min})$

\begin{tabular}{llll}
\hline Study & \multicolumn{2}{l}{ Histopathological grade } & \\
\cline { 2 - 4 } & High & Intermediate & Low grade \\
\hline Kovac et al. [24] & BF: $17.45 \pm 4.1^{\mathrm{a}}$ & & BF: $28.5 \pm 7.7^{\mathrm{a}}$ \\
& BV: $2.66 \pm 1.0^{\mathrm{a}}$ & & BV: $5.5 \pm 1.4^{\mathrm{a}}$ \\
D'Onofrio et al. [40] & BF: $5.9^{\mathrm{b}}$ & & BF: $8.9^{\mathrm{b}}$ \\
& BV: $11.3^{\mathrm{a}, \mathrm{b}}$ & & BV: $19^{\mathrm{a}, \mathrm{b}}$ \\
Schneewei $\beta$ et al. [39] & BF: $21.9 \pm 10.4$ & BF: $21.9 \pm 10.4$ & BF: $20.6 \pm 8.6$ \\
(Max Slope + Patlak) & BV: $5.5 \pm 4.5$ & BV: $5.5 \pm 4.5$ & BV: $8.9 \pm 11.3$ \\
& PS: $11.5 \pm 6.4$ & PS: $21.0 \pm 10.2$ & PS: $19.3 \pm 4.5$ \\
Schneewei $\beta$ et al. [39] & BF: $35.6 \pm 13.9$ & BF: $37.7 \pm 16.6$ & BF: $33.5 \pm 10.3$ \\
(Deconvolution) & BV: $6.1 \pm 3.9$ & BV: $7.9 \pm 5.5$ & BV: $6.4 \pm 1.3$ \\
& PS: $11.9 \pm 7.1$ & PS: $13.7 \pm 8.8$ & PS: $11.5 \pm 6.4$ \\
\hline
\end{tabular}

Kovac and D'Onofrio classified both moderate- and well-differentiated lesions as low grade ${ }^{a}$ Significant differences between pathological grading groups. Grade was high (poorly differentiated), intermediate (moderately differentiated), or low (well differentiated)

${ }^{\mathrm{b}}$ Median values, rest of the table report mean values

Table 4 Mean/median perfusion parameters of responders and Non-responders during CTP performed at baseline and follow-up after chemoradiotherapy

\begin{tabular}{lllll}
\hline Study & Baseline responder & Baseline non-responder & FU responder & FU non-responder \\
\hline Hamdy et al. [35] & BF: $(\mathrm{mL} / 100 \mathrm{~g} / \mathrm{min}): 44^{\mathrm{a}}$ & BF: $28^{\mathrm{a}}$ & ${\text { BF: } 54^{\mathrm{b}}}^{\mathrm{b}}$ & BF: 43 \\
& BV: $(\mathrm{mL} / 100 \mathrm{~g}): 4.3$ & BV: 2.0 & BV: $6.8^{\mathrm{b}}$ & BV: 4.8 \\
& PS: $(\mathrm{mL} / 100 \mathrm{~g} / \mathrm{min}): 25$ & PS: 20 & PS: 32 & PS: 28 \\
Park et al. [41] & Permeability $(\mathrm{mL} / 100 \mathrm{~mL} / \mathrm{min}):$ & Permeability: $19.0 \pm 10.9^{\mathrm{a}}$ & & \\
& $50.8 \pm 30.5^{\mathrm{a}}$ & BV: $4.1 \pm 1.7$ & & \\
& BV $(\mathrm{mL} / 100 \mathrm{~mL}): 5.7 \pm 3.0$ & & \\
\hline
\end{tabular}

Parameters of Hamdy reported as median values, parameters of Park reported as mean results

${ }^{a}$ Significant difference between responder and non-responder

${ }^{\mathrm{b}}$ Significant difference compared to baseline perfusion parameters of responders. Parameters of Hamdy reported as median values, parameters of Park reported as mean results

the fraction of contrast medium that remains in the tissue and can be calculated by deconvolution. On the basis of this impulse residue function, the BF, BV, and MTT are approximated [50-52].

Mean perfusion parameters in PDAC and non-tumorous pancreatic tissue as included in this review show a wide variance as visible in Figs. 2, 3, and 4. When studying the differences between the models, mean BF values calculated with the maximum slope model were significantly lower than using the deconvolution method $[20.4 \pm 9.7 \mathrm{~mL} / \mathrm{min} / 100 \mathrm{~g}$ and $36.9 \pm 15.6 \mathrm{~mL} / \mathrm{min} / 100 \mathrm{~g}$ $(p<0.004)$ ] [39]. BV values calculated with the deconvolution method resulted in significant higher values than with the Patlak model $[7.3 \pm 4.7 \mathrm{~mL} / 100 \mathrm{~g}$ vs. $5.6 \pm 5.5 \mathrm{~mL} / 100 \mathrm{~g}(p<0.001)]$, in contrast to the values found for PS $[12.4 \pm 8.2 \mathrm{~mL} / 100 \mathrm{~g} / \mathrm{min}$ for deconvolution vs $18.9 \pm 9.8 \mathrm{~mL} / 100 \mathrm{~g} / \mathrm{min}$ for Patlak, $(p<0.001)]$. However, comparing perfusion parameters of both models, a good correlation was found between Deconvolution and
Max slope + Patlak parameters using ICC and Pearson linear correlation coefficient [39].

\section{Risk of bias}

Figure 5 shows the results of risk of bias and concerns about applicability using the QUADAS-2 tool. Overall studies show a low risk of bias; however, the index test is not always accurately reported, especially concerning the use of the kinetic model. Some studies show an inconsistency, as reported perfusion parameters cannot be calculated from the presented kinetic model, and these studies were marked as high risk in bias for the index test.

Patient flow and timing between CTP and reference standard were reported poorly in almost all studies. For QUADAS-2 per study, see Fig. 4. 


\begin{tabular}{|c|c|c|c|c|c|c|c|}
\hline \multirow[t]{2}{*}{ Study } & \multicolumn{4}{|c|}{ RISK OF BIAS } & \multicolumn{3}{|c|}{$\begin{array}{l}\text { APPLICABILITY } \\
\text { CONCERNS }\end{array}$} \\
\hline & 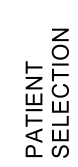 & 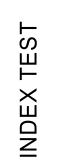 & 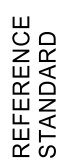 & 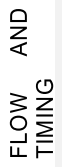 & 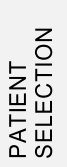 & 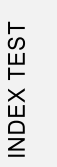 & 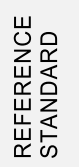 \\
\hline Aslan et al. [21] & (;) & (;) & ;) & $?$ & (:) & (;) & (;) \\
\hline Bao J. et al. [29] & (;) & $\ddot{2}$ & ;) & $?$ & (:) & (:) & (;) \\
\hline Delrue et al. [35] & (;) & (2) & ;) & $?$ & 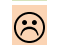 & (:) & (:) \\
\hline Delrue et al. [36] & (;) & 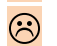 & ;) & ? & (:) & (;) & (;) \\
\hline D'Onofrio et al. [40] & (;) & (;) & (:) & (:) & (:) & (:) & (;) \\
\hline Hamdy et al. [34] & (:) & (:) & (:) & ? & (:) & (:) & (:) \\
\hline Kandel et al. [26] & (:) & (:) & (:) & ? & (:) & (:) & (:) \\
\hline Klauss et al. [27] & (;) & (:) & (:) & ? & (:) & (:) & (:) \\
\hline Konno et al. [43] & (:) & (:) & (:) & 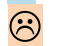 & $\ddot{\theta}$ & (:) & (:) \\
\hline Kovac et al. [23] & (:) & (:) & (:) & ? & (:) & (;) & (:) \\
\hline Li et al. [31] & (:) & 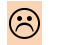 & (:) & ? & (:) & (;) & (;) \\
\hline Li et al. [25] & (:) & 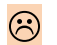 & (:) & ? & (:) & (:) & (:) \\
\hline Lu et al. [30] & (:) & 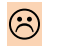 & (;) & ? & (:) & (;) & (;) \\
\hline Nishikawa et al. [42] & (;) & 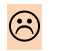 & 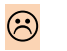 & ? & (:) & 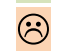 & (;) \\
\hline O'Malley et al.[24] & $\ddot{\theta}$ & (:) & 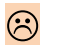 & ? & (:) & (:) & $\ddot{\theta}$ \\
\hline Park et al. [41] & (;) & (:) & (;) & ? & (:) & (:) & (:) \\
\hline Skornitzke et al. [28] & (:) & (:) & (:) & ? & (:) & (:) & (:) \\
\hline Schneewei $\beta$ et al. [39] & (:) & $\ddot{\theta}$ & (:) & ? & (:) & $\ddot{\theta}$ & (:) \\
\hline Tan et al. [33] & $\ddot{\theta}$ & (:) & (:) & ? & $\ddot{\theta}$ & (:) & (:) \\
\hline Xu et al. [22] & (:) & $\ddot{\theta}$ & (:) & ? & (:) & (:) & (:) \\
\hline Yadav et al. [32] & (:) & (:) & (:) & ? & (:) & (:) & (:) \\
\hline
\end{tabular}

Fig. 5 Quality assessment of diagnostic accuracy studies (QUADAS-2)

\section{Discussion}

\section{Main study findings}

The results from this systematic review show that CTP can accurately distinguish PDAC from non-tumorous pancreatic parenchyma using perfusion parameters calculated with a kinetic model. There is a clear consensus that PDAC can be distinguished from non-tumorous pancreatic parenchyma with a significantly lower BF and BV in PDAC compared to non-tumorous pancreatic parenchyma. Only a few studies showed a significant difference in PS between PDAC and non-tumorous pancreatic parenchyma, but the mean PS for PDAC was consistently lower. Although not studied prospectively, perfusion parameters seem to be able to improve the detection of PDAC that is visually isoattenuating on biphasic CECT.
For grading, CTP can be used as an additional imaging biomarker, which is an important prognostic variable of survival in PDAC. In two studies, a significant difference was found between high-grade tumors and low-grade tumors for $\mathrm{BV}$, and in one of these studies, $\mathrm{BF}$ also showed a significant difference $[24,40]$. In a third study, no significant difference could be found between three pathological grades using BF, $\mathrm{BV}$, and permeability [39]. There is no clear consensus for the use of CTP as a biomarker for the pathological grade. Nonetheless, two out of three studies show significant differences between grades. Large clinical studies are needed to correlate perfusion parameters to histological grade on the resection specimen.

Treatment assessment using CTP was investigated in two studies. Pre-treatment BF and permeability showed to be a good indicator of histopathological response to chemoradiotherapy [35, 41]. Furthermore, CTP was also performed to assess the effects after chemoradiation therapy. Both responders and non-responders showed an increase in blood flow and blood volume, but only the increase in responders was significant. The microenvironment of PDAC could provide an explanation for these findings, as PDAC has a stroma-rich tumor environment with high intratumoral pressure [52]. This could result in impaired perfusion, eventually impeding the delivery of oxygen and chemotherapy to tumor cells [53]. In tumors with less vessel restriction, reflected by higher baseline perfusion, chemotherapy is better able to penetrate the tissue. Both prediction and assessment of chemoradiotherapy response show promising results which reflect microenvironmental differences. Clinical studies are needed to assess the use of CTP in both baseline and followup of treatment, to evaluate CTP as biomarker in treatment assessment.

\section{Kinetic models/scan parameters}

Perfusion parameters strongly depend (up to $30 \%$ ) on the applied kinetic model and are not directly interchangeable, as shown in a variety of other cancers [51]. Of the included studies, 7 out of 21 used a deconvolution algorithm, 8 used a max-slope algorithm, and in 6 studies, a Patlak analysis was reported. Four studies reported a combination of the compartment models: Max-slope and Patlak analysis. The reported perfusion parameters display a wide range of variability between different kinetic models and perfusion parameters do not always correspond with the reported model. In six articles, BF values were described, even though the reported model was the Patlak model. An explanation could be that some software combines the Patlak and the maxslope models, using the latter to determine the BF. Furthermore, three studies report BV and two PS, although only a max-slope model was mentioned. 
In this review, three frequently used kinetic models are described; variations in these models exist, but use is not clearly reported in the literature. The assumptions of the kinetic model influence the calculated quantitative parameters differently. Maximum slope assumes a single compartment and, therefore, no venous outflow out of the tissue. The advantage of this model is the short acquisition time and the simplicity of mathematics. The latter also presents a disadvantage as the correlation between the assumptions made and true physiology is difficult. In all healthy tissue, venous outflow is present; therefore, the true $\mathrm{BF}$ is higher than calculated with this model.

The standard Patlak model quantifies the exchange between two compartments: the intravascular and extravascular compartments. This linear model assumes that the back-flux of contrast agent from the extravascular to the intravascular compartment is negligible [49]. This assumption can be applied only during the first pass of contrast agent in tissue and depends on the relative magnitude of blood flow and permeability surface area. However, as PDAC is a hypovascular tumor, the magnitude of blood flow could, therefore, be inadequate to meet this assumption [49, 54]. To take the back-flux into account, a modified Patlak model has been developed, although this non-linear model is more difficult to implement $[55,56]$.

Deconvolution uses the arterial and time-attenuation curves to calculate the impulse residue function for the tissue. The advantage is that BF, BV, and MTT can be calculated directly with a single model. It is assumed that contrast material is nondiffusible out of the vessels which is not an accurate theory, as there is leakage into the interstitial space [50].

All three models use different assumptions, which do not always accurately reflect pancreatic (tumor) tissue physiology. It is impossible to exactly modulate the kinetics of a contrast agent in tissue, though optimization for specific indications has led to numerous tracer-kinetic models [57, 58]. The preferred model depends on the desired parameters, target area as well as on the acquisition protocol. Since resolution and noise can influence the quantification, sensitivity to noise could explain the lack of consensus on the more mathematically complex parameter PS.

The chosen acquisition and analysis methods strongly influence estimated perfusion parameters. Included studies used a contrast dose in the range of $40-80 \mathrm{~mL}$, with exception of studies using an interleaved protocol in which a weight-based contrast dose was used. The amount of contrast agent volume (50 vs $100 \mathrm{~mL}$ ) does not substantially change quantitative perfusion parameters for colorectal cancer [59]. The reproducibility of CTP as assessed by Kaufmann et al. showed an interscan variability in the range of $30 \%$ [60]. Respiratory motion during scanning is one of the causes for interscan variability, and motion correction methods significantly improve the reproducibility of CTP [61]. Positioning and size of the measured region of interest (ROI) do influence the measured perfusion values. An ROI comprising the entire tumor diameter leads to more stable perfusion measurements, compared to a smaller ROI. As PDAC is known to be heterogeneous, an ROI in a single slice could introduce measurement bias. This is also highlighted in two studies reporting an increase in perfusion values towards the rim of the tumor [25, 36]. A 3D volume of interest would be a more reliable measurement and allows a better understanding of the whole tumor.

\section{Limitations}

For this systematic review, some limitations need to be addressed. First, the number of clinical studies performing CTP in PDAC is still low with too small study populations. The diagnostic accuracy of the technique is not compared to CECT in terms of diagnosis. Second, it is difficult to quantitively compare perfusion parameters of studies using different kinetic models, analysis software, ROI definitions, and scan parameters. As the diversity of the data influences the calculated parameters, no meta-analysis was performed. Third, poor reporting of applied kinetic models could hamper the interpretation of some included studies. Most studies risks of bias were high or unclear, making the summarized evidence limited.

\section{Future perspectives}

CTP provides high spatiotemporal resolution data for quantitative functional information about PDAC which could help in diagnosis, grading, and treatment prediction. The current use of CTP for PDAC is still limited mainly due to concerns regarding technique and knowledge. The amount of radiation for a CTP has been reduced due to improvements in detector efficiency and reconstruction algorithms. The additional information provided by CTP could help steer treatment decision and, therefore, seems to outweigh the risk of radiation.

There are several developments which could help to bring CTP into clinical practice. Advanced interleaved techniques make it possible to perform CTP during routine multiphase contrast-enhanced pancreatic CT using a single-contrast injection $[25,44]$. In such a 'one-stop-shop' procedure, perfusion information is acquired without extra time and cost for the patient and can be applied in oncologic imaging adding functional information. Furthermore, due to new multidetector CT scanners, larger tissue volumes can be scanned, which facilitate perfusion evaluation of the whole tumors and their surroundings.

One of the barriers limiting widespread adoption seems the lack of reference values, scan standards, and validation. 
It is difficult to compare perfusion values across different scanners, kinetic models, software, and scan parameters, limiting the use of CTP as a quantitative imaging biomarker for clinical decision making. To increase the use of CTP, some steps need to be taken as described in the EIBALL criteria [62]. First, standardization of scan protocol and reporting of CTP need to be established, enabling better comparison of studies. In addition, perfusion parameters need to be validated with pathology assessment such as microvessel density, permeability, or aggressiveness markers. The latter allows a better understanding of quantitative values aiding in treatment response prediction and, therefore, precision treatment.

Improvement of post-processing also could lead to a more reliable assessment of perfusion parameters. Compartmental and deconvolution analysis are the most widely used kinetic models; however, there is no consensus regarding applicability for abdominal oncology. In this review, we showed that results among studies are not directly comparable. Kinetic models and CTP software are often not tailored and validated for oncology. Because of the physiological differences, other perfusion models and parameters might be more useful to assess tumor characteristics or treatment response.

Novel analysis methods using artificial intelligence (AI) could help extracting diagnostic CTP information from time-intensity curves. AI can facilitate kinetic modelindependent interpretation of CTP, reducing inter-observer bias and parameter variability. Radiomics can effectively combine all CTP parameters with spatial information to guide treatment of patients with PDAC [63]. These datadriven biomarkers and their potential to improve tumor characterization and treatment assessment are increasingly investigated [63-65]. A combination of CTP and radiomic features already shows to improve the prediction of response in laryngeal cancer [66]. Furthermore, AI methods and advanced filters could reduce noise in CTP images, improving the image quality and quantitative analysis. For instance, dynamic similarity filters not only are already in use for cardiac CTP but are also in development for abdominal CTP [67]. These developments could facilitate clinical adoption and maximize impact by optimizing the analysis of CTP images regardless of acquisition and reconstruction parameters.

\section{Conclusion}

Quantitative CTP shows a potential benefit in PDAC diagnosis and can serve as a tool for pathological grading and treatment assessment; however, clinical evidence is still limited. To improve clinical use, standardized acquisition and reconstruction parameters are necessary for the interchangeability of the perfusion parameters. The use of an interleaved CTP-CECT protocol followed by post-processing and AI-supported analysis could advance the use of CTP as a predictive biomarker for PDAC.

Supplementary Information The online version contains supplementary material available at https://doi.org/10.1007/s00261-021-03190-w.

Funding Partial financial support was received from the KWF Dutch Cancer Society (KWF:12034).

\section{Declarations}

Conflict of interest The authors have no relevant financial or non-financial interests to disclose.

Open Access This article is licensed under a Creative Commons Attribution 4.0 International License, which permits use, sharing, adaptation, distribution and reproduction in any medium or format, as long as you give appropriate credit to the original author(s) and the source, provide a link to the Creative Commons licence, and indicate if changes were made. The images or other third party material in this article are included in the article's Creative Commons licence, unless indicated otherwise in a credit line to the material. If material is not included in the article's Creative Commons licence and your intended use is not permitted by statutory regulation or exceeds the permitted use, you will need to obtain permission directly from the copyright holder. To view a copy of this licence, visit http://creativecommons.org/licenses/by/4.0/.

\section{References}

1. Siegel RL, Miller KD, Fuchs HE, Jemal A (2021) Cancer Statistics, 2021. CA Cancer J Clin 71:7-33. https://doi.org/10.3322/ caac. 21654

2. Zhang L, Sanagapalli S, Stoita A (2018) Challenges in diagnosis of pancreatic cancer. World J Gastroenterol 24:2047-2060. https:// doi.org/10.3748/wjg.v24.i19.2047

3. Willett CG, Czito BG, Bendell JC, Ryan DP (2005) Locally advanced pancreatic cancer. J Clin Oncol 23:4538-4544. https:// doi.org/10.1200/JCO.2005.23.911

4. Ducreux M, Cuhna AS, Caramella C, et al (2015) Cancer of the pancreas: ESMO Clinical Practice Guidelines for diagnosis, treatment and follow-up. Ann Oncol 26:v56-v68. https://doi.org/10. 1093/annonc/mdv295

5. Prokesch RW, Chow LC, Beaulieu CF, et al (2002) Isoattenuating pancreatic adenocarcinoma at multi-detector row CT: Secondary signs. Radiology 224:764-768. https://doi.org/10.1148/radiol. 2243011284

6. Yoon SH, Lee JM, Cho JY, et al (2011) Small ( $\leq 20 \mathrm{~mm})$ pancreatic adenocarcinomas: Analysis of enhancement patterns and secondary signs with multiphasic multidetector CT. Radiology 259:442-452. https://doi.org/10.1148/radiol.11101133

7. Elsherif SB, Virarkar M, Javadi S, et al (2020) Pancreatitis and PDAC: association and differentiation. Abdom Radiol 45:13241337. https://doi.org/10.1007/s00261-019-02292-w

8. Rochefort MM, Ankeny JS, Kadera BE, et al (2013) Impact of tumor grade on pancreatic cancer prognosis: Validation of a novel TNMG staging system. Ann Surg Oncol 20:4322-4329. https:// doi.org/10.1245/s10434-013-3159-3

9. van Riet PA, Larghi A, Attili F, et al (2019) A multicenter randomized trial comparing a 25-gauge EUS fine-needle aspiration 
device with a 20-gauge EUS fine-needle biopsy device. Gastrointest Endosc 89:329-339. https://doi.org/10.1016/j.gie.2018.10. 026

10. Cros J, Raffenne J, Couvelard A, Poté N (2018) Tumor Heterogeneity in Pancreatic Adenocarcinoma. Pathobiology 85:64-71. https://doi.org/10.1159/000477773

11. Tempero MA, Malafa MP, Chiorean EG, et al (2019) NCCN Guidelines Insights: Pancreatic Adenocarcinoma, Version 1.2019. JNCCN J Natl Compr Cancer Netw 17:203-210. https://doi.org/ 10.6004/jncen.2019.0014

12. Cassinotto C, Mouries A, Lafourcade JP, et al (2014) Locally advanced pancreatic adenocarcinoma: Reassessment of response with CT after neoadjuvant chemotherapy and radiation therapy. Radiology 273:108-116. https://doi.org/10.1148/radiol.14132914

13. Wagner M, Antunes C, Pietrasz D, et al (2017) CT evaluation after neoadjuvant FOLFIRINOX chemotherapy for borderline and locally advanced pancreatic adenocarcinoma. Eur Radiol 27:3104-3116. https://doi.org/10.1007/s00330-016-4632-8

14. Miles KA, Hayball MP, Dixon AK (1995) Measurement of human pancreatic perfusion using dynamic computed tomography with perfusion imaging. Br J Radiol 68:471-475. https://doi.org/10. 1259/0007-1285-68-809-471

15. Miles KA, Griffiths MR (2003) Perfusion CT: A worthwhile enhancement? Br J Radiol 76:220-231. https://doi.org/10.1259/ bjr $/ 13564625$

16. Truong MT, Saito N, Ozonoff A, et al (2011) Prediction of locoregional control in head and neck squamous cell carcinoma with serial CT perfusion during radiotherapy. Am J Neuroradiol 32:1195-1201. https://doi.org/10.3174/ajnr.A2501

17. Yabuuchi H, Kawanami S, Iwama E, et al (2018) Prediction of therapeutic effect of chemotherapy for NSCLC using dual-input perfusion CT analysis: Comparison among bevacizumab treatment, two- agent platinum-based therapy without bevacizumab, and other non- bevacizumab treatment groups. Radiology 286:685-695. https://doi.org/10.1148/radiol.2017162204

18. Sahani D V., Kalva SP, Hamberg LM, et al (2005) Assessing tumor perfusion and treatment response in rectal cancer with multisection CT: Initial observations. Radiology 234:785-792. https://doi.org/10.1148/radiol.2343040286

19. Jiang T, Kambadakone A, Kulkarni NM, et al (2012) Monitoring response to antiangiogenic treatment and predicting outcomes in advanced hepatocellular carcinoma using image biomarkers, ct perfusion, tumor density, and tumor size (recist). Invest Radiol 47:11-17. https://doi.org/10.1097/RLI.0b013e3182199bb5

20. Liberati A, Altman DG, Tetzlaff J, et al (2009) The PRISMA statement for reporting systematic reviews and meta-analyses of studies that evaluate health care interventions: Explanation and elaboration. PLoS Med. https://doi.org/10.1371/journal.pmed. 1000100

21. Perik T, Van Genugten E, Hermans JJ, et al Quantitative CT perfusion imaging in patients with pancreatic cancer: A systematic review. In: PROSPERO Int. Prospect. Regist. Syst. Rev. https:// www.crd.york.ac.uk/prospero/display_record.php?RecordID $=$ 213438. Accessed 18 Jun 2021

22. Aslan S, Nural MS, Camlidag I, Danaci M (2019) Efficacy of perfusion $\mathrm{CT}$ in differentiating of pancreatic ductal adenocarcinoma from mass-forming chronic pancreatitis and characterization of isoattenuating pancreatic lesions. Abdom Radiol 44:593-603. https://doi.org/10.1007/s00261-018-1776-9

23. Xu J, Liang Z, Hao S, et al (2009) Pancreatic adenocarcinoma: Dynamic 64-slice helical CT with perfusion imaging. Abdom Imaging 34:759-766. https://doi.org/10.1007/s00261-009-9564-1

24. Kovač JD, Durić-Stefanović A, Dugalić V, et al (2019) CT perfusion and diffusion-weighted MR imaging of pancreatic adenocarcinoma: can we predict tumor grade using functional parameters?
Acta radiol 60:1065-1073. https://doi.org/10.1177/0284185118 812202

25. O'Malley RB, Soloff E V., Coveler AL, et al (2020) Feasibility of wide detector $\mathrm{CT}$ perfusion imaging performed during routine staging and restaging of pancreatic ductal adenocarcinoma. Abdom Radiol. https://doi.org/10.1007/s00261-020-02786-y

26. Li HO, Guo J, Sun C, et al (2015) Assessment of pancreatic adenocarcinoma: Use of low-dose whole pancreatic CT perfusion and individualized dual-energy CT scanning. J Med Imaging Radiat Oncol 59:590-598. https://doi.org/10.1111/1754-9485.12342

27. Kandel S, Kloeters C, Meyer H, et al (2009) Whole-organ perfusion of the pancreas using dynamic volume CT in patients with primary pancreas carcinoma: Acquisition technique, post-processing and initial results. Eur Radiol 19:2641-2646. https://doi.org/ 10.1007/s00330-009-1453-z

28. Klauß M, Stiller W, Fritz F, et al (2012) Computed tomography perfusion analysis of pancreatic carcinoma. J Comput Assist Tomogr 36:237-242. https://doi.org/10.1097/RCT.0b013e3182 $4 \mathrm{a} 099 \mathrm{e}$

29. Skornitzke S, Kauczor HU, Stiller W (2019) Measuring Dynamic CT Perfusion Based on Time-Resolved Quantitative DECT Iodine Maps: Comparison to Conventional Perfusion at $80 \mathrm{kVp}$ for Pancreatic Carcinoma. Invest Radiol 54:689-696. https://doi.org/10. 1097/RLI.0000000000000591

30. Bao J, Liu A, Zhao C, et al (2019) Correlation Between DualEnergy Computed Tomography Single Scan and Computed Tomography Perfusion for Pancreatic Cancer Patients: Initial Experience. J Comput Assist Tomogr 43:599-604. https://doi. org/10.1097/RCT.0000000000000878

31. Lu N, Feng XY, Hao SJ, et al (2011) 64-slice CT perfusion imaging of pancreatic adenocarcinoma and mass-forming chronic pancreatitis. Acad Radiol 18:81-88. https://doi.org/10.1016/j. acra.2010.07.012

32. Li HO, Sun C, Xu ZD, et al (2014) Low-dose whole organ CT perfusion of the pancreas: Preliminary study. Abdom Imaging 39:40-47. https://doi.org/10.1007/s00261-013-0045-1

33. Yadav AK, Sharma R, Kandasamy D, et al (2016) Perfusion CT - Can it resolve the pancreatic carcinoma versus mass forming chronic pancreatitis conundrum? Pancreatology 16:979-987. https://doi.org/10.1016/j.pan.2016.08.011

34. Tan Z, Miao Q, Li X, et al (2015) The primary study of lowdose pancreas perfusion by $640-$ slice helical CT: a wholeorgan perfusion. Springerplus 4:0-6. https://doi.org/10.1186/ s40064-015-0950-6

35. Hamdy A, Ichikawa Y, Toyomasu Y, et al (2019) Perfusion CT to assess response to neoadjuvant chemotherapy and radiation therapy in pancreatic ductal adenocarcinoma: Initial experience. Radiology 292:628-635. https://doi.org/10.1148/radiol.20191 82561

36. Delrue L, Blanckaert P, Mertens D, et al (2011) Assessment of tumor vascularization in pancreatic adenocarcinoma using 128slice perfusion computed tomography imaging. J Comput Assist Tomogr 35:434-438. https://doi.org/10.1097/RCT.0b013e3182 $23 f 0 c 5$

37. Delrue L, Blanckaert P, Mertens D, et al (2012) Tissue perfusion in pathologies of the pancreas: Assessment using 128-slice computed tomography. Abdom Imaging 37:595-601. https://doi.org/ 10.1007/s00261-011-9783-0

38. Klauß M, Stiller W, Pahn G, et al (2013) Dual-energy perfusionCT of pancreatic adenocarcinoma. Eur J Radiol 82:208-214. https://doi.org/10.1016/j.ejrad.2012.09.012

39. Schneeweiß S, Horger M, Grözinger A, et al (2016) CT-perfusion measurements in pancreatic carcinoma with different kinetic models: Is there a chance for tumour grading based on functional parameters? Cancer Imaging 16:1-8. https://doi.org/10.1186/ s40644-016-0100-6 
40. D’Onofrio M, Gallotti A, Mantovani W, et al (2013) Perfusion CT can predict tumoral grading of pancreatic adenocarcinoma. Eur J Radiol 82:227-233. https://doi.org/10.1016/j.ejrad.2012.09.023

41. Park MS, Klotz E, Kim MJ, et al (2009) Perfusion CT: Noninvasive surrogate marker for stratification of pancreatic cancer response to concurrent chemo- And radiation therapy. Radiology 250:110-117. https://doi.org/10.1148/radiol.2493080226

42. Nishikawa Y, Tsuji Y, Isoda H, et al (2014) Perfusion in the Tissue Surrounding Pancreatic Cancer and the Patient's Prognosis. Biomed Res Int 2014:648021. https://doi.org/10.1155/2014/ 648021

43. Hermans JJ (2013) Liver and Pancreatic perfusion using Aquilion ONE Vision. In: Present. Eur. Congr. Radiol. March 2013. https:// www.youtube.com/watch? $\mathrm{v}=\mathrm{rZMbJPnBPvw}$

44. Konno Y, Hiraka T, Kanoto M, et al (2020) Pancreatic perfusion imaging method that reduces radiation dose and maintains image quality by combining volumetric perfusion CT with multiphasic contrast enhanced-CT. Pancreatology 20:1406-1412. https://doi. org/10.1016/j.pan.2020.08.010

45. Cuenod CA, Balvay D (2013) Perfusion and vascular permeability: Basic concepts and measurement in DCE-CT and DCE-MRI. Diagn Interv Imaging 94:1187-1204. https://doi.org/10.1016/j. diii.2013.10.010

46. Petralia G, Bonello L, Viotti S, et al (2010) CT perfusion in oncology: How to do it. Cancer Imaging 10:8-19. https://doi.org/10. 1102/1470-7330.2010.0001

47. Dankbaar JW, Hom J, Schneider T, et al (2008) Dynamic perfusion CT assessment of the blood-brain barrier permeability: First pass versus delayed acquisition. Am J Neuroradiol 29:1671-1676. https://doi.org/10.3174/ajnr.A1203

48. Prezzi D, Khan A, Goh V (2015) Perfusion CT imaging of treatment response in oncology. Eur J Radiol 84:2380-2385. https:// doi.org/10.1016/j.ejrad.2015.03.022

49. Lee TY (2002) Functional CT: Physiological models. Trends Biotechnol 20:S3. https://doi.org/10.1016/S0167-7799(02)02035-8

50. García-Figueiras R, Goh VJ, Padhani AR, et al (2013) CT perfusion in oncologic imaging: A useful tool? Am J Roentgenol 200:8-19. https://doi.org/10.2214/AJR.11.8476

51. Niu T, Yang P, Sun X, et al (2018) Variations of quantitative perfusion measurement on dynamic contrast enhanced CT for colorectal cancer: Implication of standardized image protocol. Phys Med Biol 63:165009. https://doi.org/10.1088/1361-6560/aacb99

52. Koh TS, Bisdas S, Koh DM, Thng CH (2011) Fundamentals of tracer kinetics for dynamic contrast-enhanced MRI. J Magn Reson Imaging 34:1262-1276. https://doi.org/10.1002/jmri.22795

53. Neesse A, Michl P, Frese KK, et al (2011) Stromal biology and therapy in pancreatic cancer. Gut 60:861-868. https://doi.org/10. 1136/gut.2010.226092

54. Feig C, Gopinathan A, Neesse A, et al (2013) The pancreas cancer microenvironment. Clin Cancer Res 18:4266-4276. https://doi. org/10.1158/1078-0432.CCR-11-3114.The

55. Karakatsanis NA, Zhou Y, Lodge MA, et al (2015) Generalized whole-body patlak parametric imaging for enhanced quantification in clinical PET. Phys Med Biol 60:8643-8673. https://doi. org/10.1088/0031-9155/60/22/8643

56. Patlak CS, Blasberg RG (1985) Graphical evaluation of bloodto-brain transfer constants from multiple-time uptake data.
Generalizations. J Cereb Blood Flow Metab 5:584-590. https:// doi.org/10.1038/jcbfm. 1985.87

57. Sourbron SP, Buckley DL (2013) Classic models for dynamic contrast-enhanced MRI. NMR Biomed 26:1004-1027. https://doi. org/10.1002/nbm.2940

58. Deniffel D, Boutelier T, Labani A, et al (2018) Computed Tomography Perfusion Measurements in Renal Lesions Obtained by Bayesian Estimation, Advanced Singular-Value Decomposition Deconvolution, Maximum Slope, and Patlak Models: Intermodel Agreement and Diagnostic Accuracy of Tumor Classification. Invest Radiol 53:477-485. https://doi.org/10.1097/RLI.00000 00000000477

59. Goh V, Bartram C, Halligan S (2009) Effect of intravenous contrast agent volume on colorectal cancer vascular parameters as measured by perfusion computed tomography. Clin Radiol 64:368-372. https://doi.org/10.1016/j.crad.2008.08.018

60. Kaufmann S, Schulze M, Horger T, et al (2015) Reproducibility of VPCT Parameters in the Normal Pancreas. Comparison of Two Different Kinetic Calculation Models. Acad Radiol 22:1099_ 1105. https://doi.org/10.1016/j.acra.2015.04.005

61. Chu LL, Knebel RJ, Shay AD, et al (2018) CT perfusion imaging of lung cancer: benefit of motion correction for blood flow estimates. Eur Radiol 28:5069-5075. https://doi.org/10.1007/ s00330-018-5492-1

62. deSouza NM, Achten E, Alberich-Bayarri A, et al (2019) Validated imaging biomarkers as decision-making tools in clinical trials and routine practice: current status and recommendations from the EIBALL* subcommittee of the European Society of Radiology (ESR). Insights Imaging 10:87. https://doi.org/10. 1186/s 13244-019-0764-0

63. Tomaszewski MR, Gillies RJ (2021) The biological meaning of radiomic features. Radiology 298:505-516. https://doi.org/10. 1148/radiol.2021202553

64. Tanadini-Lang S, Bogowicz M, Veit-Haibach P, et al (2018) Exploratory radiomics in computed tomography perfusion of prostate cancer. Anticancer Res 38:685-690. https://doi.org/10. 21873/anticanres.12273

65. Bogowicz M, Tanadini-Lang S, Veit-Haibach P, et al (2019) Perfusion $\mathrm{CT}$ radiomics as potential prognostic biomarker in head and neck squamous cell carcinoma. Acta Oncol (Madr) 58:1514-1518. https://doi.org/10.1080/0284186X.2019.1629013

66. Woolen S, Virkud A, Hadjiiski L, et al (2021) Prediction of Disease Free Survival in Laryngeal and Hypopharyngeal Cancers Using CT Perfusion and Radiomic Features: A Pilot Study. Tomography 7:10-19. https://doi.org/10.3390/tomography70100 02

67. Kouchi T, Tanabe Y, Smit EJ, et al (2020) Clinical application of four-dimensional noise reduction filtering with a similarity algorithm in dynamic myocardial computed tomography perfusion imaging. Int J Cardiovasc Imaging 36:1781-1789. https://doi.org/ 10.1007/s10554-020-01878-6

Publisher's Note Springer Nature remains neutral with regard to jurisdictional claims in published maps and institutional affiliations. 